\title{
ON THE HOMOTOPY GROUPS OF $A(X)$
}

\author{
STANISLAW BETLEY
}

\begin{abstract}
In this paper we will prove that if $X$ is any space with a finite fundamental group, then Waldhausen's algebraic $K$-groups of $X$ are finitely generated. We will use Dwyer's machinery developed in Twisted homological stability for general linear groups (Ann. of Math. 111).
\end{abstract}

Introduction. In [D] Dwyer proved the following theorem:

THEOREM (DWYER). If $X$ is simply-connected and $\pi_{i}(X)$ is finitely generated for $i \geq 2$, then $\pi_{j}(A(X))$ is finitely generated for all $j$, where $A(X)$ is Waldhausen's algebraic $K$-theory of a space $X$.

In this paper we will use Dwyer's approach in order to generalize his theorem to the case when $\pi_{1}(X)$ is a finite group. We will prove the following theorem.

THEOREM I. If $\pi_{1}(X)$ is a finite group and $\pi_{i}(X)$ is finitely generated for $i \geq 2$, then $\pi_{j}(A(X))$ is finitely generated for all $j$.

REMARK. Theorem $I$ is, in general, the best possible result in this direction. Consider $X=S^{1}$. Then using a spectral sequence $E_{p, q}^{2} \Rightarrow \pi_{p+q}(A(X))$ (for details see [W, Proposition 2.6]) where

(i) $E_{p, q}^{2}=\pi_{p+q}\left(\operatorname{fiber}\left(A^{q-1}(X) \rightarrow A^{q-2}(X)\right)\right)$ if $q \geq 2$,

(ii) $E_{p, q}^{2}=\pi_{p+1} K(Z[G(X)])$ if $q=1$

and where $\cdots \rightarrow A^{j}(X) \rightarrow \cdots$ is a tower derived from the Postnikow tower for $\Omega^{\infty} S^{\infty}$, it is easy to see that $\pi_{2}\left(A\left(S^{1}\right)\right)$ contains an infinite number of copies of $Z / 2 Z$ so $\pi_{2}\left(A\left(S^{1}\right)\right)$ is not finitely generated.

I. Let $R$ be a ring and let $A$ be an $R$-bimodule which is finitely generated as an abelian group. Then $M_{n} A$-the abelian group of $(n \times n)$-matrices with entries in $A$-is an $M_{n} R$-bimodule and $M_{n} A$ becomes a left $\mathrm{Gl}_{n} R$-module by defining

$$
\begin{aligned}
g \circ m=g m g^{-1} & \text { for } m \in M_{n} A, g \in \mathrm{Gl}_{n} R \text { where the multiplication } \\
& \text { is taken with respect to the } R \text {-bimodule } \\
& \text { structure on } A .
\end{aligned}
$$

Now let $A^{n}$ be a left $\mathrm{Gl}_{n} R$-module with the obvious action. Consider $\bar{R}^{n}$-the left $\mathrm{Gl}_{n} R$-module which is isomorphic to $R^{n}$ as an abelian group and where the action of $\mathrm{Gl}_{n} R$ is given by

$$
g x\left(r_{1}, \ldots, r_{n}\right)=\left(r_{1}, \ldots, r_{n}\right) g^{-1} \text { for all } g \in \mathrm{Gl}_{n} R .
$$

Received by the editors July 19, 1985.

1980 Mathematics Subject Classification. Primary 55D99, 55B99, $18 \mathrm{H} 10$.

$K e y$ words and phrases. Algebraic $K$-theory of a space, Postnikow tower. 
The abelian group $\bar{R}^{n}$ is also a left $R$-module with the obvious action and these two left module structures on $\bar{R}^{n}$ commute:

$$
\begin{aligned}
g x\left(r\left(r_{1}, \ldots, r_{n}\right)\right) & =g x\left(r r_{1}, \ldots, r r_{n}\right)=\left(r r_{1}, \ldots, r r_{n}\right) g^{-1} \\
& =r\left(\left(r_{1}, \ldots, r_{n}\right) g^{-1}\right)=r\left(g x\left(r_{1}, \ldots, r_{n}\right)\right)
\end{aligned}
$$

for $g \in \mathrm{Gl}_{n} R, r \in R,\left(r_{1}, \ldots, r_{n}\right) \in \bar{R}^{n}$.

Proposition 1.3. The $\mathrm{Gl}_{n} R$-module $M_{n} A$ given by (1.1) is isomorphic to $A^{n} \otimes_{R} \bar{R}^{n}$, where $A^{n} \otimes_{R} \bar{R}^{n}$ is a left $\mathrm{Gl}_{n} R$-module by the diagonal action.

Proof. We have the map $F: A^{n} \otimes_{R} \bar{R}^{n} \rightarrow M_{n} A$ given by

$$
\left(a_{1}, \ldots, a_{n}\right) \otimes\left(r_{1}, \ldots, r_{n}\right) \stackrel{F}{\longrightarrow}\left|\begin{array}{lc}
a_{1} r_{1}, \ldots, a_{1} r_{n} \\
\vdots & \vdots \\
a_{n} r_{1}, \ldots, a_{n} r_{n}
\end{array}\right| .
$$

This is obviously a group isomorphism. It is easy to see by computing both sides that for any $g \in \mathrm{Gl}_{n} R,\left(a_{1}, \ldots, a_{n}\right) \in A^{n}$, and $\left(r_{1}, \ldots, r_{n}\right) \in \bar{R}^{n}$ we have

$$
g \circ F\left(\left(a_{1}, \ldots, a_{n}\right) \otimes\left(r_{1}, \ldots, r_{n}\right)\right)=F\left(g\left(a_{1}, \ldots, a_{n}\right) \otimes g x\left(r_{1}, \ldots, r_{n}\right)\right),
$$

so $F$ is a $\mathrm{Gl}_{n} R$ isomorphism of modules.

In the following proposition we will use van der Kallen's definitions and notation from $[\mathbf{K}]$ (see also [D]):

Proposition 1.4. Let $T:\left(\mathrm{Gl}_{n} R \text {-left modules }\right)^{2} \rightarrow A b$ be a functor of finite degree. Then $T\left(A^{n}, \bar{R}^{n}\right)$ is a strongly stable system for $\mathrm{Gl}_{n} R$.

Proof. Do precisely the same as in the proof of Dwyer's Lemma 3.1 in [D].

We can obtain immediately the following corollary from Proposition 1.4.

COROLLARY 1.5. In the notation from (1.1) and (1.3), $M_{n} A$ is a strongly stable system of coefficients for $\mathrm{Gl}_{n} R$.

II. Now we are able to prove Theorem I. Let us recall (see [W, $\$ 2]$ for details) that $A(X)$ is an $H$-space with the identity component given by $\widehat{B G l}(X)^{+}$, so Theorem I follows easily from the following lemma (see [D, Lemma 4.1]):

LEMMA 2.1. If $X$ is as in Theorem I, then the integral homology groups of $\widehat{B G l}(X)$ are finitely generated.

Before we prove Lemma 2.1 let us make the following remark.

REMARK 2.2. The properties of $\widehat{B G l}(X)$ which we need are

(i) $\pi_{1}(\widehat{B G l}(X))=\operatorname{Gl}(Z \pi)$, where $\pi=\pi_{1}(X)$;

(ii) $\pi_{k}(\widehat{B G l}(X))$ is isomorphic as a $\mathrm{Gl}(Z \pi)$-module to

$$
M\left(\pi_{k-1}^{s}(\Omega X \cup \mathrm{pt})\right)=\lim _{n} M_{n}\left(\pi_{k-1}^{s}(\Omega X \cup \mathrm{pt})\right)
$$

with the action given by (1.1).

Let $\mathfrak{M}$ be the smallest class of $\operatorname{Gl}(Z \pi)$-modules which has the following properties:

(1) $\mathfrak{M}$ contains $M(A)$ for all $Z \pi$-bimodules $A$, which are finitely generated as abelian groups; 
(2) $\mathfrak{M}$ contains all finitely generated abelian groups with the trivial $\pi$-action;

(3) If $\mathfrak{M}$ contains $A$ and $B$, then $\mathfrak{M}$ contains $H_{j}(K(A, n) ; B)$ for $n \geq 2$ and $j \geq 0$.

We will need the following lemma.

LEMMA 2.3. If $A \in \mathfrak{M}$, then the groups $H_{j}(\mathrm{Gl}(Z \pi) ; A)$ are finitely generated.

Proof OF LEMMA 2.1 FROM LEMMA 2.3. Let $P_{k} \widehat{B G l}(X)$ be a $k$ th stage of the Postnikow system for $\widehat{B G l}(X)$. We will show by induction on $k$ that for any integer $k$ and $A \in \mathfrak{M}$ the homology groups $H_{j}\left(P_{k} \widehat{B G l}(X) ; A\right)$ are finitely generated.

(i) If $k=1$, then this is exactly Lemma 2.3 .

(ii) Let $k>1$. Look at the fibration

$$
K\left(\pi_{k}(\widehat{B G l}(X)), k\right) \rightarrow P_{k}(\widehat{B G l}(X)) \rightarrow P_{k-1}(\widehat{B G l}(X))
$$

Then for any $A \in \mathfrak{M}$ we have the Serre spectral sequence with twisted coefficients:

$$
E_{p, q}^{2}=H_{p}\left(P_{k-1}(\widehat{B G l}(X)) ; H_{q}\left(K\left(\pi_{k}(\widehat{B G l}(X)), k\right) ; A\right)\right)
$$

converging to $H_{p+q}\left(P_{k}(\widehat{B G l}(X)) ; A\right)$.

But $\pi_{k}(\widehat{B G l}(X))=M\left(\pi_{k-1}^{s}(\Omega X \cup \mathrm{pt})\right) \in \mathfrak{M}$, so $H_{q}\left(K\left(\pi_{k}(\widehat{B G l}(X)), k\right) ; A\right) \in \mathfrak{M}$ and by the induction hypothesis all groups in $E_{* *}^{2}$ are finitely generated. This gives us immediately that for all $j, H_{j}\left(P_{k}(\widehat{B G l}(X)) ; A\right)$ are finitely generated.

Now we will need two more lemmas (see [D, Lemmas 4.3 and 3.2]).

LEMMA 2.4. The functor $T: A b^{2} \rightarrow A b$ given by $T(A, B)=H_{j}(K(A, n) ; B)$, $n \geq 2, j \geq 0$, is of finite degree with respect to $A$ and $B$.

Proof. Precisely the same as the proof of Dwyer's Lemma 4.3.

LEMMA 2.5. Let $A, B, C$ be abelian categories and let $S: A \rightarrow B$ and $T: B \rightarrow C$ be functors. If $S$ has degree $k$ and $T$ has degree l, then the composite $T S$ has degree $\leq k l$.

Now we are able to prove Lemma 2.3 which will finish the proof of Theorem I. We will need two simple observations:

2.6. If $\pi$ is a finite group, then $\mathrm{Gl}_{n}(Z \pi)$ is an arithmetic group. This is the special case of the much more general situation (see [S, Example 5]): if $R$ is a ring which is finitely generated as a $Z$-module, then $R^{*}$ is an arithmetic group. In our case take $R=M_{n}(Z \pi)$.

2.7. If $\pi$ is a finite group, then van der Kallen's stability theorems with twisted coefficients hold for $Z \pi$ (see [K] for details).

Every module in the class $\mathfrak{M}$ can be expressed as $T(M(A))$, where $A$ is finitely generated over $Z$ and $T$ is a functor of finite degree which commutes with direct limits and preserves the subcategory of finitely generated abelian groups. Then

$$
T(M(A))=\lim _{n} T\left(M_{n}(A)\right)
$$

and

$$
H_{j}(\mathrm{Gl}(Z \pi) ; T(M(A)))=\lim _{n} H_{j}\left(\mathrm{Gl}_{n}(Z \pi) ; T\left(M_{n}(A)\right)\right)
$$


By Proposition 1.4 and Lemma 2.5, $T\left(M_{n}(A)\right)$ is a strongly stable system for $\mathrm{Gl}_{n}(Z \pi)$ so

$$
\lim _{n} H_{j}\left(\mathrm{Gl}_{n}(Z \pi) ; T\left(M_{n}(A)\right)\right)=H_{j}\left(\mathrm{Gl}_{N}(Z \pi) ; T\left(M_{N}(A)\right)\right)
$$

for sufficiently large $N$ (by (2.7)). In [R] Raghunathan proved that for an arithmetic group $G$ and a $Z G$-module $M$ which is finitely generated as an abelian group, $H_{j}(G ; M)$ is finitely generated for all $j \geq 0$. So by observation $(2.6)$ and $[\mathbf{R}]$ we know that the homology groups $H_{j}\left(\mathrm{Gl}_{N}(Z \pi) ; T\left(M_{N}(A)\right)\right)$ are finitely generated. This completes the proof of Lemma 2.3.

ACKNOWLEDGEMENT. I would like to thank Bill Dwyer for many helpful conversations and encouragement during my work on this paper.

\section{REFERENCES}

[D] W. G. Dwyer, Twisted homological stability for general linear groups, Ann. of Math. 111 (1980), 239-251.

[K] W. van der Kallen, Homology stability for linear groups, Invent. Math. 60 (1980), $269-295$.

[R] M. S. Raghunathan, A note on the quotients of real algebraic groups by arithmetic subgroups, Invent. Math. 4 (1968), 318-335.

[S] J.-P. Serre, Arithmetic groups, Homological Group Theory, (C. T. C. Wall, ed.), Cambridge Univ. Press, 1979, pp. 105-136.

[W] F. Waldausen, Algebraic K-theory of topological spaces. I, Proc. Sympos. Pure Math., vol. 32, Part 1, Amer. Math. Soc., Providence, R.I., 1978, pp. 35-60.

Department of Mathematics, Universitiy of Notre Dame, Notre Dame, InDIANA 46556

Current address: Instytut Matematyki, Wydzial Matematyki UW, PKiN, IXp, 00-901 Warszawa, Poland 